\title{
Effects of Position and Force of Calf Compression with Graded Ankle Plantar Flexion on Central Aponeurosis Displacement
}

\author{
TAKAHIRo OTSUdo ${ }^{1)}$, Hitoshi TAKeI ${ }^{2)}$, ATSUSHI SENOO $^{3)}$ \\ ${ }^{1)}$ Department of Physical Therapy, Graduate School of Human Health Sciences of Tokyo \\ Metropolitan University: 7-2-10, Higashiogu, Arakawa City, Tokyo 116-8551, Japan. \\ TEL:+813-3819-1211,E-mail: otsudo@saitama-med.ac.jp \\ ${ }^{2)}$ Division of Physical Therapy, Faculty of Health Sciences, Tokyo Metropolitan University \\ ${ }^{3)}$ Division of Radiological Sciences, Faculty of Health Sciences, Tokyo Metropolitan University
}

J. Phys. Ther. Sci.

22: $35-41,2010$

\begin{abstract}
Purpose] This study investigated the effects of position and force of calf compression with graded ankle plantar flexion on central aponeurosis displacement. [Subjects] Ten healthy adult men participated in this study. [Methods] The subjects were placed in the prone position with knee and ankle joint angles of $0^{\circ}$ and asked to perform graded isometric plantar flexion at $0,5,10$ or $15 \mathrm{~kg}$. The position of compression was either proximal or distal to the maximum circumference of the calf. Force of compression was set at $0,50,100$ or $150 \mathrm{mmHg}$. Under these conditions, displacement of the central aponeurosis of the gastrocnemius lateralis, soleus and triceps surae-Achilles tendon junction was measured by ultrasonography. [Results] With isometric contraction, central aponeurosis displacement of the gastrocnemius lateralis and soleus with 100 or $150 \mathrm{mmHg}$ of distal compression was significantly lower than at $0 \mathrm{mmHg}$ of distal compression. However, with proximal compression, central aponeurosis displacement with isometric contraction did not change in relation to the compression force. [Conclusion] This study showed that even when the level of compression remains unchanged, compression achieves different effects depending on the position of compression.
\end{abstract}

Key words: Aponeurosis displacement, Compression, Ultrasonography

(This article was submitted Aug. 24, 2009, and was accepted Oct. 2, 2009)

\section{INTRODUCTION}

Delayed onset muscle soreness and swelling occurring after vigorous exercise induce conditions such as restricted joint mobility, lowering competitive performance ${ }^{1)}$, and the ability to perform activities of daily living. In physical therapy, applying a proper level of compression using stockings or elastic bandages to the affected areas can occasionally improve such symptoms. Previous studies have documented that compression improves symptoms by reducing swelling ${ }^{2,3}$, increasing venous flow rate ${ }^{4}$, increasing the level of oxygenated haemoglobin ${ }^{5)}$, and decreasing lactic acid levels ${ }^{6)}$. Compression forces used in these studies were low, at $5.2-15.4 \mathrm{mmHg}^{2,3)}, 2-30$ $\mathrm{mmHg}^{4)}, 5-24.1 \mathrm{mmHg}^{5)}$ and $12.75-33 \mathrm{mmHg}^{6}$, and compression facilitated peripheral circulation in the legs and improved poor circulation following vigorous exercise.

Knee orthoses and taping techniques are commonly used for trauma-induced motor dysfunction, to reduce the load on the musculoskeletal system in the affected area ${ }^{7-9}$. Compression forces in these procedures have been estimated as $50 \mathrm{mmHg}^{7)}$, which is much greater 


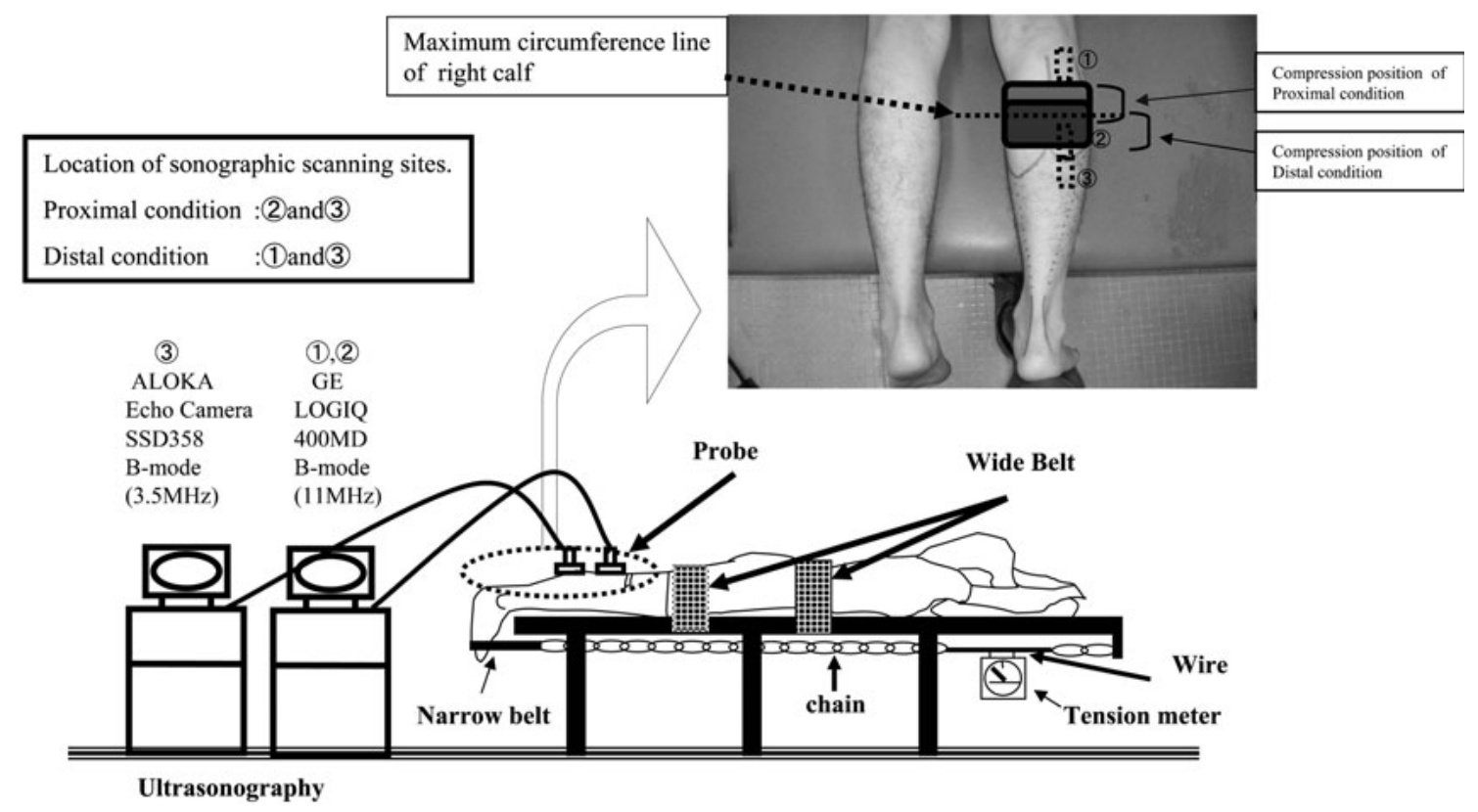

Fig. 1. Measurement set-up.

Schematic diagram of the testing equipment with a subject positioned prone on the device with femoral and lower trunk regions securely strapped to the bed. Subjects lay prone with the knee extended and the ankle at $90^{\circ}$. To determine ankle plantar flexion force, a tension meter was attached between the narrow belt and the steel frame by way of chain under the bed.

than those generated by the use of stockings or elastic bandages. Additionally, compression using a knee orthosis or taping not only reduces external load on the musculoskeletal system, but also improves neuromuscular control ${ }^{7)}$, stimulates proprioception $^{8)}$ and increases muscle recruitment ${ }^{9)}$ to instantly improve muscle force. Thus, there are various effects produced by using different apparatus, each of which has different levels of compression force. However, many issues remain unknown in terms of the effects and mechanisms of action of relatively high levels of compression created by knee orthoses and taping on muscles. There are two reasons for this: compression force is not graded, and it is not possible to identify which degree of compression will bring about changes in muscle force; and as the width and placement of orthoses has varied between studies, it has not been possible to determine which aspect of the abovementioned mechanisms improved muscle force.

To clarify which of these mechanisms is effective for compression in vivo, the present study ascertained the effects of force and position of calf compression with respect to isometric ankle plantar contraction using ultrasonography(US) to measure the displacement of the central aponeurosis of the soleus, gastrocnemius lateralis, and triceps suraeAchilles tendon junction.

\section{SUBJECTS AND METHODS}

The subjects were 10 healthy male volunteers (mean (standard deviation) age, 24.6 (5.3) years; height, 170.6 (4.3) cm; weight $63.3(11.9) \mathrm{kg}$ ) who participated in this study only after providing their written informed consent. The study was conducted in accordance with the Declaration of Helsinki and all protocols were approved by the ethics committee of Tokyo Metropolitan University (No. 05084).

Measurements were made at the right gastrocnemius lateralis, soleus and triceps suraeAchilles tendon junction, and subjects did not have a history of orthopaedic disorders in these areas or the right ankle. During testing, subjects were placed in the prone position with the knee in full extension and the ankle in a neutral position. Isometric plantar flexion was achieved using chains, a wire (diameter, $2 \mathrm{~mm}$ ) and a narrow belt (non-stretching; diameter, $24 \mathrm{~mm}$ ). The belt was placed under the bed along a line connecting the 
centre of the ball of the big toe and the centre of the ball of the little toe to achieve isometric plantar flexion. To prevent the measurement sites from moving, the trunk and thigh were immobilised using belts (non-stretching; width, $250 \mathrm{~mm}$ ) (Fig. 1). Plantar flexion was set at the following four grades: $0 \mathrm{~kg}$ (rest); $5 \mathrm{~kg} ; 10 \mathrm{~kg}$; and $15 \mathrm{~kg}$. A tension meter (weight, $360 \mathrm{~g}$; Yagami Co Ltd, Japan) was used to verify the desired levels of contraction. Based on the study by Kawakami et al. ${ }^{10)}$, subjects were instructed to maintain each level of isometric plantar flexion for 2-4 s. The order of plantar flexion was as follows: $5 \mathrm{~kg}$; $10 \mathrm{~kg}$; and $15 \mathrm{~kg}$. For compression, a cuff (Welchallyn, New York, USA) and a sphygmomanometer (Yamasu Co Ltd, Japan) were used. As to the position of compression, the centre of the width of the cuff $(10 \mathrm{~cm})$ was placed either $2 \mathrm{~cm}$ proximal or $2 \mathrm{~cm}$ distal to the maximum circumference of the right calf (Fig. 1). Compression force was set at the following four levels: $0 \mathrm{mmHg}$; $50 \mathrm{mmHg}$; $100 \mathrm{mmHg}$; and 150 $\mathrm{mmHg}$. The compression force was confirmed before each test. Each level of compression force was applied for no more than $15 \mathrm{~s}$, and subjects were given enough time to rest between measurements. Distal compression was applied first, followed by proximal compression. The order of the compression force was randomised.

Measurement of the aponeurosis and triceps surae-Achilles tendon junction displacement is possible by US in vivo. Ever since Griffiths used US to observe the skeletal muscles in vivo ${ }^{11)}$, this technique has been widely performed to assess muscle shape during muscle contraction ${ }^{12-14)}$. Studies have reported that US can measure displacement of the central aponeurosis and myotendinous junction ${ }^{15-17)}$. Displacement of the central aponeurosis and triceps surae-Achilles tendon junction also correlate proportionally to isometric contraction ${ }^{18,19)}$ with high reproducibility ${ }^{18,20)}$.

The following items were measured simultaneously using two US machines: displacement of the central aponeurosis of the gastrocnemius lateralis (GL-apo); displacement of the central aponeurosis of the soleus (SO-apo); and displacement of the triceps surae-Achilles tendon junction (musculotendinous junction (MTJ)). A GE LOGIQ400MD (B-mode, $11 \mathrm{MHz}$ ) was used to measure GL-apo and SO-apo, and an ALOKA Echo Camera SSD358 (B-mode, $3.5 \mathrm{MHz}$ ) was used to measure MTJ. Prior to the study, the US (GE LOGIQ400MD) location of gastrocnemius lateralis was marked on the skin. Measurements were made while always ensuring that the position of the probe was unchanged in relation to the skin mark. The probe was placed as gently as possible to obtain scans using gel, and was not forcibly pressed against the skin. In studies conducted by Muramatsu et al. ${ }^{18)}$ and Kuno et al. ${ }^{20)}$, aponeurosis displacement was measured based on the displacement of contact points between the aponeurosis and fascicles as measured by US. In the present study, the central aponeurosis and fascicles of the soleus and gastrocnemius lateralis were first confirmed. Next, of the various points of contact between the aponeurosis and fascicles, the points with sufficient luminance were used to measure GL-apo displacement (contact points between the central aponeurosis and fascicles of gastrocnemius lateralis) and $\mathrm{SO}$-apo displacement (contact points between central aponeurosis and fascicles of soleus). According to studies by Muramatsu et al. ${ }^{18)}$, MTJ was defined as the contact point between the distal area of gastrocnemius lateralis and the insertion of the Achilles tendon as measured by US. Muscle contraction causes GLapo, SO-apo and MTJ to shift left, and displacements to the left and right were thus given plus and minus values, respectively (Tables 1,2, 3). All ultrasonographic scans during measurement were recorded on S-VHS tape and replayed. Using the track ball and displacement measurement mode of the US, three measurements were made, and the average of these three measurements was analysed.

Two-way ANOVA was performed to analyse differences in measurements in relation to compression force at rest $(0 \mathrm{~kg}$ of isometric contraction), then a multiple comparison test (LSD method) and paired t-test were used. One-way ANOVA was also performed for each isometric contraction force $(5,10$ and $15 \mathrm{~kg})$, then the multiple comparison test (LSD method) was conducted. All statistical analysis was performed using SPSS (SPSS Inc., Chicago, USA) for Windows version 12 and significance was chosen as $\mathrm{p}<0.05$.

\section{RESULTS}

For changes in GL-apo, SO-apo and MTJ due to proximal compression force with $0 \mathrm{~kg}$ of isometric 
Table 1. Displacement of central aponeurosis (GL-apo,SO-apo) and myotendinous junction(MTJ) by compression at rest

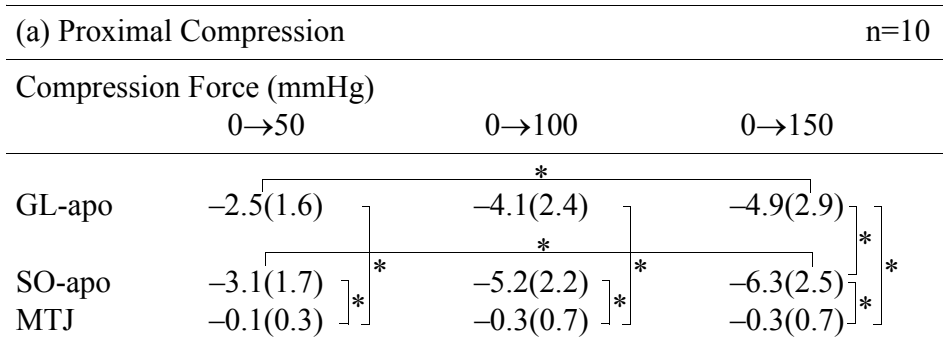

(b) Distal Compression

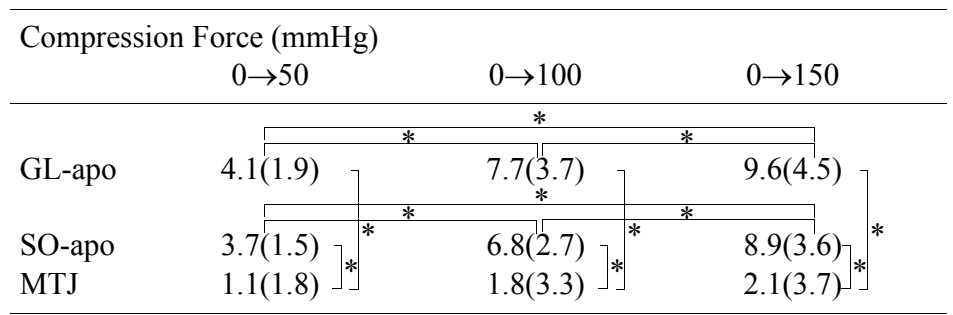

Values are mean (SD) $[\mathrm{mm}]$.

contraction (rest), two-way ANOVA showed significant differences, and a significant main effect was noted in intra-subject variations in relation to compression force. A multiple comparison test on GL-apo and SO-apo showed significant displacements in distal direction between 0 and 50 $\mathrm{mmHg}$ and between 50 and $150 \mathrm{mmHg}$. A paired ttest on compression force showed a significant difference in GL-apo and SO-apo between 0 and $150 \mathrm{mmHg}$ and in MTJ and GL-apo and SO-apo between 0 and $50 \mathrm{mmHg}$, between 0 and 100 $\mathrm{mmHg}$ and between 0 and $150 \mathrm{mmHg}$. Multiple comparisons revealed no significant displacement in MTJ in relation to compression force (Table 1a). In terms of changes in GL-apo, SO-apo and MTJ due to distal compression force with $0 \mathrm{~kg}$ of isometric contraction (rest), two-way ANOVA confirmed significant differences, and a significant main effect was documented in intra-subject variations in relation to compression force. A multiple comparison test showed significant distal displacement in GL-apo and SO-apo between 0 and $50 \mathrm{mmHg}$, between 0 and $100 \mathrm{mmHg}$, between 0 and $150 \mathrm{mmHg}$, between 50 and $100 \mathrm{mmHg}$ and between 100 and $150 \mathrm{mmHg}$. A paired t-test of compression force showed significant differences in MTJ in relation to GL-apo or SO-apo between 0 and $50 \mathrm{mmHg}$, between 0 and $100 \mathrm{mmHg}$ and between 0 and $150 \mathrm{mmHg}$. Multiple comparisons revealed no significant displacement in MTJ in relation to compression force (Table 1b).

With regard to changes in GL-apo with isometric contraction in relation to compression position and force, one-way ANOVA on isometric contraction showed no significant difference with proximal compression (Table 2a). However, with distal compression, multiple comparison testing showed that compared to $0 \mathrm{mmHg}, \mathrm{GL}-a p o$ was significantly decreased by 100 and $150 \mathrm{mmHg}$ at all levels of isometric contraction $(5,10$ and $15 \mathrm{~kg}$ ) (Table 2b).

For changes in SO-apo with isometric contraction in relation to compression position and force, oneway ANOVA on isometric contraction showed no significant difference with proximal compression (Table 3a). However, with distal compression, multiple comparison testing showed that SO-apo was significantly lower at $50 \mathrm{mmHg}$ of compression than at 100 or $150 \mathrm{mmHg}$ of compression with 15 $\mathrm{kg}$ of isometric contraction. With 100 or 150 $\mathrm{mmHg}$ of compression, SO-apo was significantly lower at all levels of isometric contraction $(5,10$ and $15 \mathrm{~kg}$ ) (Table 3b).

As far as changes in MTJ in relation to compression position and force with isometric contraction were concerned, one-way ANOVA on isometric contraction showed no significant difference induced by proximal or distal compression. 
Table 2. Displacement of GL central aponeurosis by compression during graded isometric contraction

\begin{tabular}{lcccc}
\hline \multicolumn{2}{l}{ (a) Proximal Compression } & $\mathrm{n}=10$ \\
\hline \multicolumn{2}{l}{ Muscle Force $(\mathrm{Kg})$} & \multicolumn{4}{c}{ Compression Force $(\mathrm{mmHg})$} \\
& 0 & 50 & 100 & 150 \\
\hline 5 & $24.3(5.2)$ & $22.9(5.4)$ & $22.9(5.1)$ & $23.3(5.5)$ \\
10 & $27.5(5.2)$ & $25.4(4.8)$ & $25.9(5.0)$ & $26.0(5.4)$ \\
15 & $28.3(4.6)$ & $29.6(4.7)$ & $26.5(3.9)$ & $27.6(3.2)$ \\
\hline
\end{tabular}

\begin{tabular}{|c|c|c|c|c|}
\hline \multirow[t]{2}{*}{ Muscle Force (Kg) } & \multicolumn{4}{|c|}{ Compression Force $(\mathrm{mmHg})$} \\
\hline & 0 & 50 & 100 & 150 \\
\hline & & $*$ & & \\
\hline 5 & $24.3(5.2)$ & $22.3(4.2)$ & $19.6(3.9)$ & $18.9(3.3)$ \\
\hline 10 & $27.5(5.2)$ & $\frac{*}{23.6(4.0)}$ & $21.5(3.1)$ & $20.1(3.4)$ \\
\hline 15 & $28.3(4.6)$ & $\frac{*}{23.4(4.0)}$ & $22.8(3.6)$ & $20.8(2.8)$ \\
\hline
\end{tabular}

Values are mean (SD) [mm].

Table 3. Displacement of SO central aponeurosis by compression during graded isometric contraction

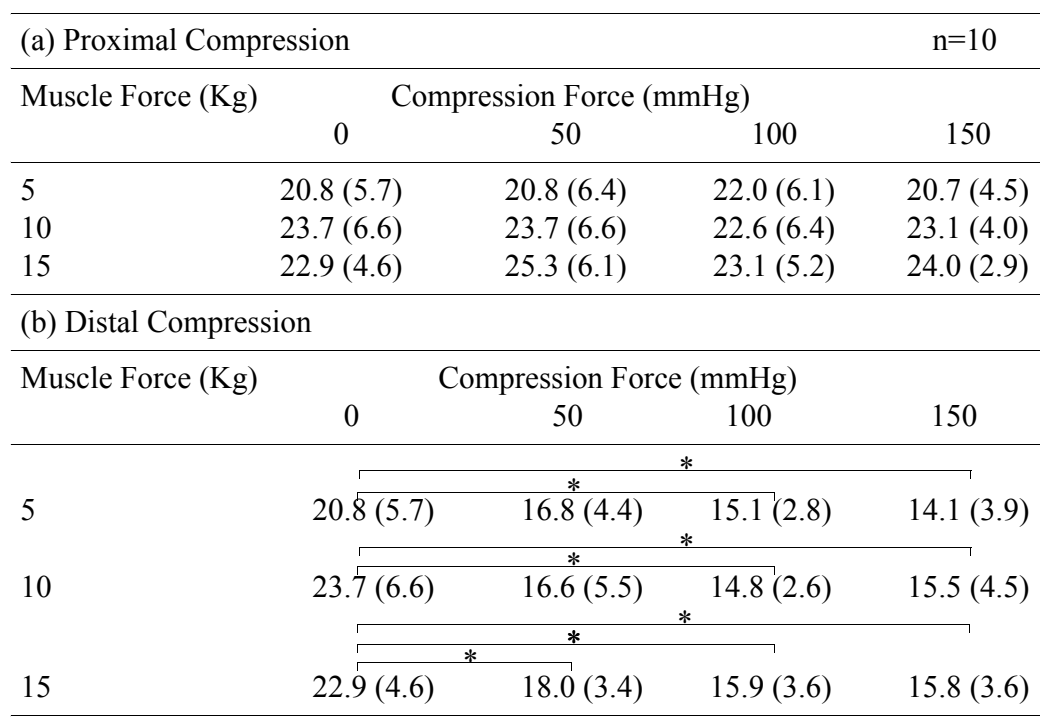

Values are mean (SD) [mm].

\section{DISCUSSION}

The present results show that GL-apo and SO-apo with distal isometric contraction $(5,10$ and $15 \mathrm{~kg})$ at 100 or $150 \mathrm{mmHg}$ of compression force were significantly smaller than those without compression $(0 \mathrm{mmHg}$ ) (Tables $2 \mathrm{~b}, 3 \mathrm{~b})$. This was because distal compression with $0 \mathrm{~kg}$ of isometric contraction displaced GL-apo and SO-apo in the proximal direction (Table $1 \mathrm{~b}$ ), matching the direction of GL-apo and SO-apo displacement with isometric contraction. In other words, isometric contraction and compression worked cooperatively for GL-apo and SO-apo displacement at all levels of isometric contraction. Compression significantly reduced the load (force $\times$ distance) at all levels of 
isometric contraction. The present results also showed that even with more than $100 \mathrm{mmHg}$ of compression, proximal compression did not result in any significant changes to GL-apo or SO-apo at all levels of isometric contraction.

Past studies have reported that compression increases muscle force by improving neuromuscular control $^{7)}$, improving proprioception ${ }^{8)}$ and increasing recruitment ${ }^{9}$. However, none of those studies directly examined these mechanisms, and only suggested their involvement in improving muscle force.

Miyamoto et al. ${ }^{21)}$ reported that increased intramuscular pressure improved stiffness of the trunk. Intramuscular pressure has been quantified by inserting a pressure gauge between muscle fibres and measuring hydrostatic pressure inside muscles with or without muscle contraction. Studies have documented that intramuscular pressure with maximum torque was $75-100 \mathrm{mmHg}$ for the biceps brachii $^{22)}$, about $200 \mathrm{mmHg}$ for the tibialis anterior and soleus ${ }^{23)}$, and $479 \mathrm{mmHg}$ for the quadriceps femoris $^{24)}$. Intramuscular pressure is proportional to isometric muscle force ${ }^{24,25)}$, and some studies have suggested that increased intramuscular pressure improves muscle force ${ }^{25}$ ). However, the suggestion that increased intramuscular pressure improves muscle force does not appear rational. This is because the distribution of intramuscular pressure over the entire muscle tissue is not even, being high at the tendon at both ends compared to the central region ${ }^{26)}$. In other words, an increase in overall intramuscular pressure cannot be concluded to improve muscle force. Furthermore, intramuscular pressure reportedly increases to curve muscle fibres as muscle force improves ${ }^{24,26)}$, but intramuscular pressure resulting in such curvature can also be deduced to work to prevent muscle fibre-shortening during muscle contraction.

Our results indicate that the central aponeurosis displacement was different at the same compression force when the compression position was different. Thus, we conclude that not only force but also the site of compression is very important for reducing the load at all levels of isometric contraction by working contraction force and compression force cooperatively.

When applying the results of the present study to clinical settings, because individual differences may exist in relationships between compression force and central aponeurosis displacement, the proper level of compression must be ascertained by US for each patient. In addition, since compression is applied to move the central aponeurosis in the direction of muscle contraction, therapists must identify the target muscles, and understand the shape of each muscle and the direction of central aponeurosis displacement due to contraction.

\section{REFERENCES}

1) Clarkson PM, Nosaka K, Braun B: Muscle function after exercise-induced muscle damage and rapid adaptation. Med Sci Sports Exerc, 1992, 24: 512-520.

2) Kraemer WJ, Volek JS, Bush JA, et al.: Influence of compression hosiery on physiological responses to standing fatigue in women. Med Sci Sport Exerc, 2000, 32: 1849-1858.

3) Kraemer WJ, Bush JA, Wickham RB, et al. : Continuous compression as an effective therapeutic intervention in treating eccentric-exercise-induced muscle soreness. J Sport Rehabil, 2001, 10: 11-23.

4) Lawrence D, Kakkar VV: Graduated static external compression of the lower limb: a physiological assessment. Br J Surg, 1980, 67: 119-121.

5) Bringard A, Denis R, Belluye N, et al:: Effects of compression tights on calf muscle oxygenation and venous pooling during quiet resting in supine and standing positions. J Sports Med Phys Fitness, 2006, 46: 548-554.

6) Chatard JC, Atlaoui D, Farjanel J, et al.: Elastic stockings, performance and leg pain recovery in 63year-old sportsmen. Eur J Appl Physiol, 2004, 93: 347-352.

7) Styf J: The effects of functional knee bracing on muscle function and performance. Sports Med, 1999, 28: 77-81.

8) Van TD, Witvrouw E, Roget P, et al.: Effect of bracing on the prevention of anterior knee Pain - a prospective randomized study. Knee Surg Sports Traumatol Arthosc, 2004, 12: 434-439.

9) Robbins S, Waked E, Rappel R: Ankle taping improves proprioception before and after exercise in young men. Br J Sports Med, 1995, 29: 242-247.

10) Kawakami $Y$, Muraoka $T$, Ito $S$, et al.: In vivo muscle fiber behaviour during counter-movement exercise in humans reveals a significant role for tendon elasticity. J Physiol, 2002, 540: 635-646.

11) Griffiths RI: Ultrasound transit time gives direct measurement of muscle fibre length in vivo. J Neurosci Methods, 1987, 21: 159-165.

12) Reeves ND, Narici MV: Behavior of human muscle fascicles during shortening and lengthening contractions in vivo. J Appl Physiol, 2003, 95: 10901096.

13) Hodges PW, Pengel LH, Herbert RD, et al.: Measurement of muscle contraction with ultrasound 
imaging. Muscle Nerve, 2003, 27: 682-692.

14) Narici MV, Binzoni $T$, Hiltbrand $E$, et al.: In vivo human gastrocnemius architecture with changing joint angle at rest and during graded isometric contraction. $\mathrm{J}$ Physiol, 1996, 496: 287-297.

15) Fukashiro $S$, Itoh $M$, Ichinose $Y$, et al.: Ultrasonography gives directly but noninvasively elastic characteristic of human tendon in vivo. Eur J Appl Physiol, 1995, 71: 555-557.

16) Maganaris CN, Paul JP: In vivo human tendon mechanical properties. J Physiol (Lond), 1999, 521: 307-313.

17) Kubo $\mathrm{K}$, Yata $\mathrm{H}$, Kanehisa $\mathrm{H}$, et al.: Effects of isometric squat training on the tendon stiffness and jump performance. Eur J Appl Physiol, 2006, 96: 305314.

18) Muramatsu $T$, Muraoka $T$, Takeshita $D$, et al.: Mechanical properties of tendon and aponeurosis of human gastrocnemius muscle in vivo. J Appl Physiol, 2001, 90: 1671-1678.

19) Maganaris CN, Paul JP: Load-elongation characteristics of in vivo human tendon and aponeurosis. J Exp Biol, 2000, 203: 751-756.

20) Kuno S, Fukunaga T: Measurement of muscle fibre displacement during contraction by real-time ultrasonography in humans. Eur J Physiol, 1995, 70: 45-48.

21) Miyamoto K, Iinuma N, Maeda M, et al.: Effects of abdominal belts on intra-abdominal pressure, intramuscular pressure in the erector spinae muscles and myoelectrical activities of trunk muscles. Clinical Biomech, 1999, 14: 79-87.

22) Korner L, Parker P, Almstrom C, et al.: Relation of intramuscular pressure to the force output and myoelectric signal of skeletal muscle. J Ortho Res, 1984, 2: 289-296.

23) Aratow M, Ballard RE, Crenshaw AG, et al.: Intramuscular pressure and electromyography as indexes of force during isokinetic exercise. J Appl Physiol, 1993, 74: 2634-2640.

24) Van Leeuwen JL, Spoor CW: Modelling mechanically stable muscle architectures. Phil Trans R Soc Lond, 1992, 336: 275-292.

25) Davis J, Kaufman KR, Lieber RL: Correlation between active and passive isometric force and intramuscular pressure in the isolated rabbit tibialis anterior muscle. $\mathrm{J}$ Biomech, 2003, 36: 505-512.

26) Otten E: Concepts and models of functional architecture in skeletal muscle. Exerc Sport Sci Rev, 1988, 16: 89-137. 